\title{
Regiochemical Control in the Metal-Catalyzed Transposition of Allylic Silyl Ethers
}

Eric C. Hansen, Daesung Lee*

Department of Chemistry, University of Wisconsin, Madison, WI 53706

\section{Supporting Information}

\section{Materials and Methods}

All compounds were purchased from Aldrich and used without purification unless otherwise indicated. $\mathrm{RuCp}\left(\mathrm{CH}_{3} \mathrm{CN}\right)_{3} \mathrm{PF}_{6}$ was purchased from Strem Chemicals, Inc. Acetone was distilled from $\mathrm{MgSO}_{4}$. Dichloromethane was distilled from $\mathrm{CaH}_{2}$. Flash chromatography was performed using silica gel $60 \AA$ (32-63 mesh) from Sorbent Technologies. Reactions were monitored by thin layer chromatography (TLC) using 0.25 mm E. Merck precoated silica gel 60 (particle size $0.040-0.063 \mathrm{~mm}$ ). ${ }^{1} \mathrm{H}$ NMR and ${ }^{13} \mathrm{C}$ NMR spectra were recorded on a Bruker AC-300 spectrometer. Chemical shifts are reported in parts per million (ppm) values relative to the internal standard tetramethyl silane (TMS). Multiplicities were reported as follows: singlet (s), doublet (d), triplet (t), quartet (q), quintet (quint), multiplet (m), broad (b). The reported NMR data is for the major isomer. High resolution mass spectra (ESI) were provided by the University of Wisconsin-Madison mass spectrometry facility. 
General procedure for Alder-ene reaction: A solution of TBS-protected 4-propene-2ol (50 mg, $0.25 \mathrm{mmol}$ ) in $2 \mathrm{~mL}$ dry acetone was added 4,4,5,5-tetramethyl-2-(prop-1ynyl)-1,3,2-dioxaborolane ${ }^{1}$ (42 $\mathrm{mg}, 0.25 \mathrm{mmol}$ ) and the solution degassed by bubbling $\mathrm{N}_{2}$ for $10 \mathrm{~min}$. $\mathrm{RuCp}\left(\mathrm{CH}_{3} \mathrm{CN}\right)_{3} \mathrm{PF}_{6}(10 \mathrm{mg}, 0.023 \mathrm{mmol})$ was added and the reaction stirred at room temperature until no further conversion of alkene is detected by TLC (1-2h). After this time, the solvent is evaporated and the residue purified by silica gel chromatography (Hex:Et $\left.{ }_{2} \mathrm{O}, 20: 1\right)$, giving $5 \mathrm{mg}$ of recovered alkene and $66 \mathrm{mg}(72 \%)$ 1e as a colorless oil.

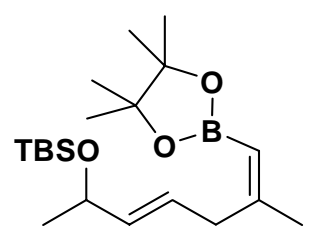

1e: ${ }^{1} \mathbf{H}$ NMR (300 MHz, $\left.\mathrm{CDCl}_{3}\right) \delta 5.51$ (m, 2H), 5.14 (s, 1H), 4.26 (m, 1H), $3.11\left(\mathrm{abq}, J_{\mathrm{ab}}=14.8 \mathrm{~Hz}, 1 \mathrm{H}\right), 3.07$ (abq, $J_{\mathrm{ab}}=14.8$ Hz, 1H), 1.82 (d, $1.3 \mathrm{~Hz}, 3 \mathrm{H}), 1.25$ (s, 12H), 1.19 (d, $J=6.6 \mathrm{~Hz}$, 3H), 0.89 (s, 9H), 0.05 (s, 3H), 0.04 (s, 3H); ${ }^{13} \mathrm{C}$ NMR (75 MHz, $\left.\mathrm{CDCl}_{3}\right) \delta 161.37,136.20,127.37,82.82,69.56,39.30,26.49,26.15,25.05,24.84,18.54$, -4.28, -4.52; HRMS (ESI) calcd for $\mathrm{C}_{20} \mathrm{H}_{39} \mathrm{BO}_{3} \mathrm{Si} \quad[\mathrm{M}+\mathrm{Na}]^{+}$: 389.2659, found 389.2666.

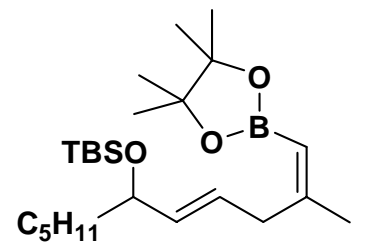

5a: ${ }^{1} \mathbf{H}$ NMR (300 MHz, $\left.\mathrm{CDCl}_{3}\right) \delta 5.46(\mathrm{~m}, 2 \mathrm{H}), 5.15(\mathrm{~s}, 1 \mathrm{H})$, 4.04 (m, 1H), 3.10 (d, $J=5.8 \mathrm{~Hz}, 2 \mathrm{H}), 1.82$ (d, $J=1.1 \mathrm{~Hz}, 3 \mathrm{H})$, 1.46 (m, 2H), 1.26 (m, 6H), 1.25 (s, 12H), 0.88 (bs, 12H), 0.03 (s, 3H), 0.01 (s, 3H); $\left.{ }^{13} \mathrm{C} \mathrm{NMR} \mathrm{(75} \mathrm{MHz,} \mathrm{CDCl}_{3}\right) \delta 161.33,135.25$, 128.36, 82.80, 73.89, 45.31, 39.36, 38.70, 32.04, 26.16, 25.06, 22.88, 21.27, 18.50, 14.25, -3.96, -4.54; HRMS (ESI) calcd for $\mathrm{C}_{24} \mathrm{H}_{47} \mathrm{BO}_{3} \mathrm{Si}[\mathrm{M}+\mathrm{Na}]^{+}$: 445.3285, found 445.3273 .

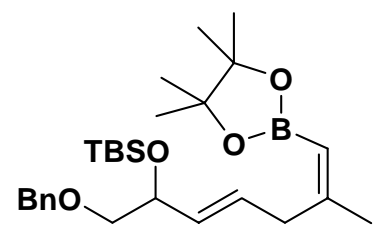

5b: ${ }^{1} \mathbf{H}$ NMR $\left(300 \mathrm{MHz}, \mathrm{CDCl}_{3}\right) \delta 5.63(\mathrm{dt}, J=15.4,6.7 \mathrm{~Hz}$, 1H), 5.51 (dd, $J=15.4,9.6 \mathrm{~Hz}, 1 \mathrm{H}), 5.15$ (d, $J=1.4 \mathrm{~Hz}, 1 \mathrm{H}$ ), 4.54 (abq, $\left.J_{\mathrm{ab}}=13.3 \mathrm{~Hz}, 2 \mathrm{H}\right), 4.29$ (bq, $\left.J=6.0 \mathrm{~Hz}, 1 \mathrm{H}\right), 3.41$ $\left(\mathbf{a b x}, J_{\mathrm{ab}}=9.7 \mathrm{~Hz}, J_{\mathrm{ax}}=7.4 \mathrm{~Hz}, 1 \mathrm{H}\right), 3.39\left(\mathrm{abx}, J_{\mathrm{ab}}=9.7 \mathrm{~Hz}, J_{\mathrm{bx}}\right.$ = 4.5 Hz, 1H), 3.13 (d, $J=6.8 \mathrm{~Hz}, 2 \mathrm{H}), 1.82$ (d, $J=1.3 \mathrm{~Hz}, 3 \mathrm{H}), 1.24$ (s, 12H), 0.89 (s, 9H), 0.06 (s, 3H), 0.04 (s, 3H); ${ }^{13}$ C NMR (75 MHz, $\left.\mathrm{CDCl}_{3}\right) \delta$ 161.09, 138.83, 131.77, 130.1, 128.47, 127.72, 127.60, 82.82, 75.55, 73.50, 72.87, 39.46, 26.49, 29.11, 25.04,

\footnotetext{
${ }^{1}$ Brown, H. C.; Bhat, N. G.; Srebnik, M. Tetrahedron Lett. 1988, 29, 2631.
} 
18.53, -4.30, -4.49; HRMS (ESI) calcd for $\mathrm{C}_{27} \mathrm{H}_{45} \mathrm{BO}_{4} \mathrm{Si} \quad[\mathrm{M}+\mathrm{Na}]^{+}$: 495.3078, found 495.3072 .

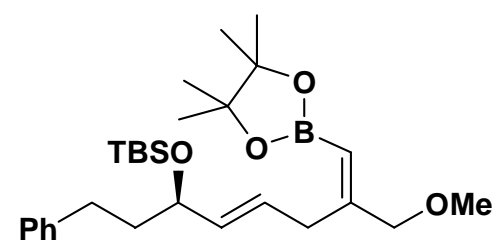

5c: ${ }^{1} \mathbf{H}$ NMR $\left(300 \mathrm{MHz}, \mathrm{CDCl}_{3}\right) \delta 7.24(\mathrm{~m}, 2 \mathrm{H}), 7.15(\mathrm{~m}$, 3H), 5.52 (m, 2H), 5.43 (s, 1H), 4.08 (q, $J=5.9 \mathrm{~Hz}, 1 \mathrm{H}$ ), 3.85 (d, $J=1.2 \mathrm{~Hz}, 2 \mathrm{H}$ ), 3.21 (s, 3H), 3.11 (d, $J=5.4 \mathrm{~Hz}$, 2H), $2.61(\mathrm{~m}, 2 \mathrm{H}), 1.77$ (m, 2H), 1.24 (s, 12H), 0.88 (s, 9H), 0.02 (s, 3H), 0.0 (s, 3H); ${ }^{13}$ C NMR (75 MHz, $\left.\mathrm{CDCl}_{3}\right) \delta$ 159.36, 142.75, 134.82, 128.72, 128.58, 128.49, 125.82, 83.06, 76.41, 73.23, 58.41, 40.38, 34.92, 31.88, 26.15, 25.04, 18.47, -3.89, -4.52; HRMS (ESI) calcd for $\mathrm{C}_{28} \mathrm{H}_{47} \mathrm{BO}_{4} \mathrm{Si} \quad[\mathrm{M}+\mathrm{Na}]^{+}$: 509.3234, found 509.3244 .

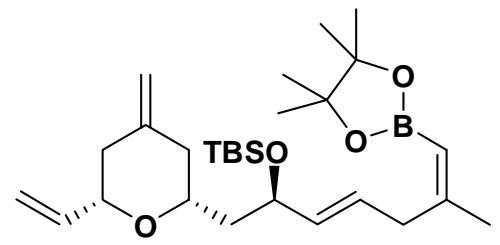

5d: ${ }^{1} \mathbf{H}$ NMR $\left(300 \mathrm{MHz}, \mathrm{CDCl}_{3}\right) \delta 5.88$ (ddd, $J=17.3$, 10.7, $4.7 \mathrm{~Hz}, 1 \mathrm{H}$ ), 5.49 (m, 2H), 5.25 (bd, $J=17.3 \mathrm{~Hz}$, 1H), 5.14 (bs, 1H), 5.10 (dt, $J=10.7,1.7 \mathrm{~Hz}, 1 \mathrm{H}), 4.72$ (bs, 2H), 4.31 (m, 1H), 3.71 (m, 1H), 3.53 (m, 1H), 3.10 (d, $J=5.5 \mathrm{~Hz}, 2 \mathrm{H}$ ), 2.24 (m, 2H), 1.95 (m, 4H), 1.81 (bs, 3H), 1.24 (s, 12H), 0.87 (s, 9H), 0.05 (s, 3H), 0.02 (s, 3H); ${ }^{13}$ C NMR (75 MHz, $\left.\mathrm{CDCl}_{3}\right) \delta$ 161.15, 144.66, 139.11, 134.40, 129.25, 114.79, 108.85, 82.82, 78.79, 75.65, 71.04, 44.96, 40.85, 39.34, 26.56, 25.07, 18.42, -3.88, -4.53; HRMS (ESI) calcd for $\mathrm{C}_{28} \mathrm{H}_{49} \mathrm{BO}_{4} \mathrm{Si} \quad[\mathrm{M}+\mathrm{Na}]^{+}$: 511.3391, found 511.3374 .

General procedure for allylic [1,3]-transposition: To a flame-dried vile under $\mathrm{N}_{2}$ atmosphere was added 5a (51 mg, $0.12 \mathrm{mmol})$ in $\sim 2 \mathrm{~mL}$ solvent $\left(\mathrm{CH}_{2} \mathrm{Cl}_{2}\right.$ or $\left.\mathrm{Et}_{2} \mathrm{O}\right)$. $\mathrm{Re}_{2} \mathrm{O}_{7}$ (3 mg, $0.006 \mathrm{mmol}$ ) was added under $\mathrm{N}_{2}$ atmosphere (glove bag). The reaction was stirred at room temperature and monitored by TLC. After $2 \mathrm{~h}$, the solvent is removed and the residue purified by silica gel chromatography (Hex:EtOAc, 5:1-2:1). The cyclic boronic acids obtained could be identified by crude ${ }^{1} \mathrm{H}$ NMR, however, quality spectra were obtained only after further derivitization. 
General procedure for Suzuki coupling: A solution of 6a ( $20 \mathrm{mg}, \sim 0.1 \mathrm{mmol})$ and (Z)-ethyl 3-iodoacrylate (28 mg, $0.12 \mathrm{mmol}$ ) in $1 \mathrm{~mL} \mathrm{THF} / \mathrm{H}_{2} \mathrm{O}$ (3:1) was degassed by bubbling $\mathrm{N}_{2}$ through the solution for $10 \mathrm{~min}$. $\mathrm{Pd}\left(\mathrm{PPh}_{3}\right)_{4}(6 \mathrm{mg}, 0.005 \mathrm{mmol})$ was added and the reaction stirred $5 \mathrm{~min}$. TlOEt (45 $\mathrm{mg}, 0.18 \mathrm{mmol}$ ) was then added giving immediate precipitation of yellow salts. After $1 \mathrm{~h}$ the reaction was diluted with ether and filtered through a silica plug. The organic layer was washed with brine and dried over $\mathrm{MgSO}_{4}$. The solvent was removed in vacuo and the residue purified by silica gel chromatography.

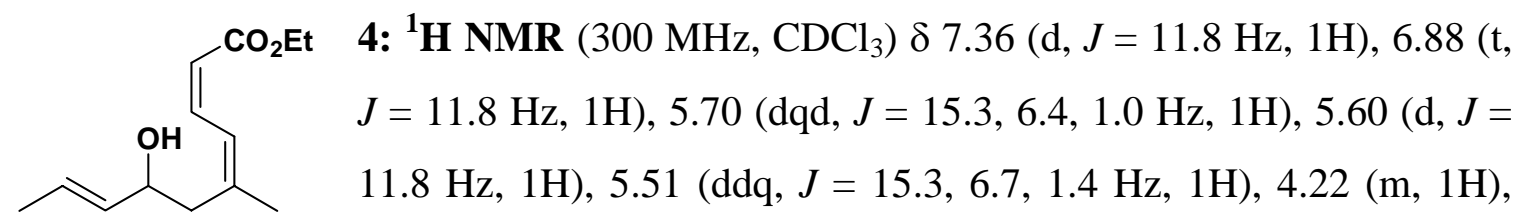
4.18 (q, $J=7.1 \mathrm{~Hz}, 2 \mathrm{H}$ ), 2.62 (dd, $J=13.2,8.3 \mathrm{~Hz}, 1 \mathrm{H}$ ), 2.33 (dd, $J=13.2,5.2 \mathrm{~Hz}, 1 \mathrm{H}$ ), 1.96 (s, 3H), 1.70 (d, $J=6.3 \mathrm{~Hz}, 3 \mathrm{H}), 1.56$ (bs, $1 \mathrm{H}), 1.29$ (t, $J=7.1 \mathrm{~Hz}, 3 \mathrm{H}) ;{ }^{13} \mathbf{C} \mathbf{N M R}$ $\left(75 \mathrm{MHz}, \mathrm{CDCl}_{3}\right) \delta 166.95,146.08,140.16,133.41,127.43,124.88,116.07,71.54$, 60.04, 40.53, 25.68, 17.85, 14.53; HRMS (ESI) calcd for $\mathrm{C}_{13} \mathrm{H}_{20} \mathrm{O}_{3} \quad[\mathrm{M}+\mathrm{Na}]^{+}:$247.1310, found 247.1300.

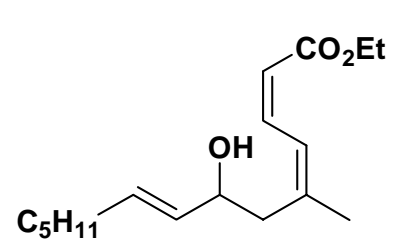

7a: ${ }^{1} \mathbf{H}$ NMR $\left(300 \mathrm{MHz}, \mathrm{CDCl}_{3}\right) \delta 7.36(\mathrm{~d}, J=11.8 \mathrm{~Hz}, 1 \mathrm{H})$, $6.88(\mathrm{t}, J=11.8 \mathrm{~Hz}, 1 \mathrm{H}), 5.68(\mathrm{dt}, J=15.8,6.7 \mathrm{~Hz}, 1 \mathrm{H}), 5.59$ (d, $J=11.8 \mathrm{~Hz}, 1 \mathrm{H}), 5.48$ (dd, $J=15.8,6.6 \mathrm{~Hz}, 1 \mathrm{H}), 4.22$ (m, 1H), 4.18 (q, $J=7.0 \mathrm{~Hz}, 2 \mathrm{H}), 2.61$ (dd, $J=13.3,8.1 \mathrm{~Hz}, 1 \mathrm{H}$ ), 2.35 (dd, $J=13.3,5.3 \mathrm{~Hz}, 1 \mathrm{H}), 2.00$ (m, 2H), 1.96 (s, 3H), 1.40-1.24 (m, 6H), 1.29 (t, $J=$ $7.0 \mathrm{~Hz}, 3 \mathrm{H}), 0.88$ (t, $J=7.0 \mathrm{~Hz}, 3 \mathrm{H}) ;{ }^{13} \mathrm{C} \mathbf{N M R}\left(75 \mathrm{MHz}, \mathrm{CDCl}_{3}\right) \delta 166.93,146.08$, 140.18, 132.81, 132.00, 124.85, 116.03, 71.59, 60.01, 40.60, 32.32, 31.57, 28.91, 25.68, 22.70, 14.51, 14.21; HRMS (ESI) calcd for $\mathrm{C}_{17} \mathrm{H}_{28} \mathrm{O}_{3} \quad[\mathrm{M}+\mathrm{Na}]^{+}$: 303.1936, found 303.1951.

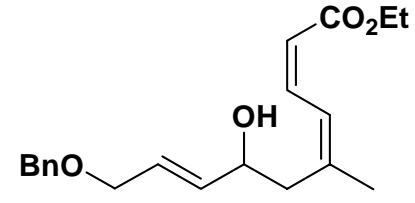

7b: ${ }^{1} \mathbf{H}$ NMR (300 MHz, $\left.\mathrm{CDCl}_{3}\right) \delta 7.39$ (d, $\left.J=11.6 \mathrm{~Hz}, 1 \mathrm{H}\right)$, 7.33 (m, 5H), 6.87 (t, $J=11.6 \mathrm{~Hz}, 1 \mathrm{H}), 5.82$ (m, 2H), 5.60 (d, $J$ $=11.6 \mathrm{~Hz}, 1 \mathrm{H}), 4.52(\mathrm{~s}, 2 \mathrm{H}), 4.32(\mathrm{~m}, 1 \mathrm{H}), 4.17$ (q, $J=7.3 \mathrm{~Hz}$, 
2H), 4.03 (bd, $J=4.3 \mathrm{~Hz}, 2 \mathrm{H}$ ), 2.63 (dd, $J=13.2,8.4 \mathrm{~Hz}, 1 \mathrm{H}$ ), 2.37 (dd, $J=13.2,4.9 \mathrm{~Hz}$, 1H), 1.97 (s, 3H), 1.29 (t, $J=7.3 \mathrm{~Hz}, 3 \mathrm{H}) ;{ }^{13} \mathbf{C}$ NMR (75 MHz, $\left.\mathrm{CDCl}_{3}\right) \delta$ 166.90, 145.59, 139.97, 138.44, 134.82, 128.62, 127.93, 127.86, 125.14, 116.35, 72.59, 70.76, 70.19, 60.07, 40.35, 25.61, 14.53; HRMS (ESI) calcd for $\mathrm{C}_{20} \mathrm{H}_{26} \mathrm{O}_{4}[\mathrm{M}+\mathrm{Na}]^{+}$: 353.1729, found 353.1742 .

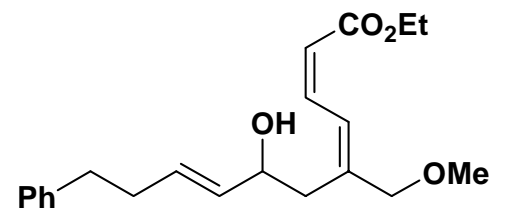

7c: ${ }^{1} \mathbf{H}$ NMR $\left(300 \mathrm{MHz}, \mathrm{CDCl}_{3}\right) \delta 7.51(\mathrm{~d}, J=11.8 \mathrm{~Hz}$, 1H), $7.28(\mathrm{~m}, 2 \mathrm{H}), 7.18(\mathrm{~m}, 3 \mathrm{H}), 6.85(\mathrm{t}, J=11.8 \mathrm{~Hz}$, 1H), 5.73 (d, $J=11.8 \mathrm{~Hz}, 1 \mathrm{H}), 5.73(\mathrm{dt}, J=15.5,6.9$ $\mathrm{Hz}, 1 \mathrm{H}$ ), 5.51 (dd, $J=15.5,6.7 \mathrm{~Hz}, 1 \mathrm{H}$ ), 4.19 (q, $J=7.0$ Hz, 2H), 4.19 (m, 1H), 4.03 (abq, $J=12.0 \mathrm{~Hz}, 1 \mathrm{H}$ ), 3.96 (abq, $J=12.0 \mathrm{~Hz}, 1 \mathrm{H}$ ), 3.37 (s, 3H), 2.81 (d, $J=3.3 \mathrm{~Hz}, 1 \mathrm{H}), 2.69$ (m, 2H), 2.51 (m, 2H), 2.35 (m, 2H), 1.30 (t, $J=7.0$ $\mathrm{Hz}, 3 \mathrm{H}) ;{ }^{13} \mathrm{C}$ NMR $\left(75 \mathrm{MHz}, \mathrm{CDCl}_{3}\right) \delta 166.50,144.24,141.92,139.02,133.00,131.27$, 128.62, 128.50, 126.07, 118.84, 77.83, 72.05, 60.25, 58.45, 37.48, 35.69, 34.17, 14.48; HRMS (ESI) calcd for $\mathrm{C}_{21} \mathrm{H}_{28} \mathrm{O}_{4}[\mathrm{M}+\mathrm{Na}]^{+}$: 367.1885, found 367.1894.

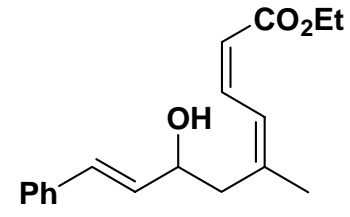

7e: ${ }^{1} \mathbf{H}$ NMR (300 MHz, $\left.\mathrm{CDCl}_{3}\right) \delta 7.40$ (d, $\left.J=11.7 \mathrm{~Hz}, 1 \mathrm{H}\right), 7.32$ (m, 5H), $6.91(\mathrm{t}, J=11.7 \mathrm{~Hz}, 1 \mathrm{H}), 6.62(\mathrm{~d}, J=16.0 \mathrm{~Hz}, 1 \mathrm{H}), 6.22$ (dd, $J=16.0,6.5 \mathrm{~Hz}, 1 \mathrm{H}$ ), 5.61 (d, $J=11.7 \mathrm{~Hz}, 1 \mathrm{H}), 4.48$ (m, $1 \mathrm{H}$ ), 4.17 (q, $J=7.0 \mathrm{~Hz}, 2 \mathrm{H}$ ), 2.73 (dd, $J=13.5,8.4 \mathrm{~Hz}, 1 \mathrm{H}$ ), 2.45 (dd, $J=13.5,5.4 \mathrm{~Hz}, 1 \mathrm{H}), 2.00$ (s, 3H), 1.29 (t, $J=7.0 \mathrm{~Hz}, 3 \mathrm{H}) ;{ }^{13} \mathrm{C}$ NMR (75 MHz, $\left.\mathrm{CDCl}_{3}\right)$ $\delta$ 166.91, 145.53, 139.72, 136.71, 131.51, 130.65, 128.81, 127.96, 126.71, 125.19, 116.38, 71.43, 60.07, 40.60, 25.72, 14.52; HRMS (ESI) calcd for $\mathrm{C}_{18} \mathrm{H}_{22} \mathrm{O}_{3} \quad[\mathrm{M}+\mathrm{Na}]^{+}$: 309.1467, found 309.1478.

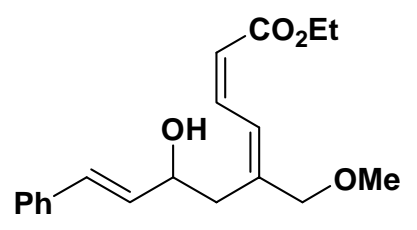

7f: ${ }^{1} \mathbf{H}$ NMR (300 MHz, $\left.\mathrm{CDCl}_{3}\right) \delta 7.54(\mathrm{~d}, J=11.8 \mathrm{~Hz}, 1 \mathrm{H})$, 7.31 (m, 5H), 6.90 (t, $J=11.8 \mathrm{~Hz}, 1 \mathrm{H}), 6.63$ (d, $J=15.8 \mathrm{~Hz}$, 1H), 6.23 (dd, $J=15.8,6.2 \mathrm{~Hz}, 1 \mathrm{H}), 5.73$ (d, $J=11.8 \mathrm{~Hz}$, $1 \mathrm{H}), 4.43(\mathrm{~m}, 1 \mathrm{H}), 4.19(\mathrm{q}, J=7.0 \mathrm{~Hz}, 2 \mathrm{H}), 4.07$ (abq, $J=$ $12.5 \mathrm{~Hz}, 1 \mathrm{H}), 4.01(\mathrm{abq}, J=12.5 \mathrm{~Hz}, 1 \mathrm{H}), 3.39(\mathrm{~s}, 3 \mathrm{H}), 2.67\left(\mathrm{abx}, J_{\mathrm{ab}}=13.8, J_{\mathrm{ax}}=4.2\right.$ $\mathrm{Hz}, 1 \mathrm{H}), 2.60$ (abx, $\left.J_{\mathrm{ab}}=13.8, J_{\mathrm{bx}}=8.3 \mathrm{~Hz}, 1 \mathrm{H}\right), 1.30(\mathrm{t}, J=7.0 \mathrm{~Hz}, 1 \mathrm{H}) ;{ }^{13} \mathbf{C}$ NMR $(75$ $\left.\mathrm{MHz}, \mathrm{CDCl}_{3}\right) \delta 166.50,143.83,138.81,1136.88,131.82,130.29,128.75,127.82$, 126.69, 126.40, 119.18, 77.91, 72.04, 60.27, 58.50, 37.56, 14.47; HRMS (ESI) calcd for $\mathrm{C}_{19} \mathrm{H}_{24} \mathrm{O}_{4}[\mathrm{M}+\mathrm{Na}]^{+}: 339.1572$, found 339.1559. 

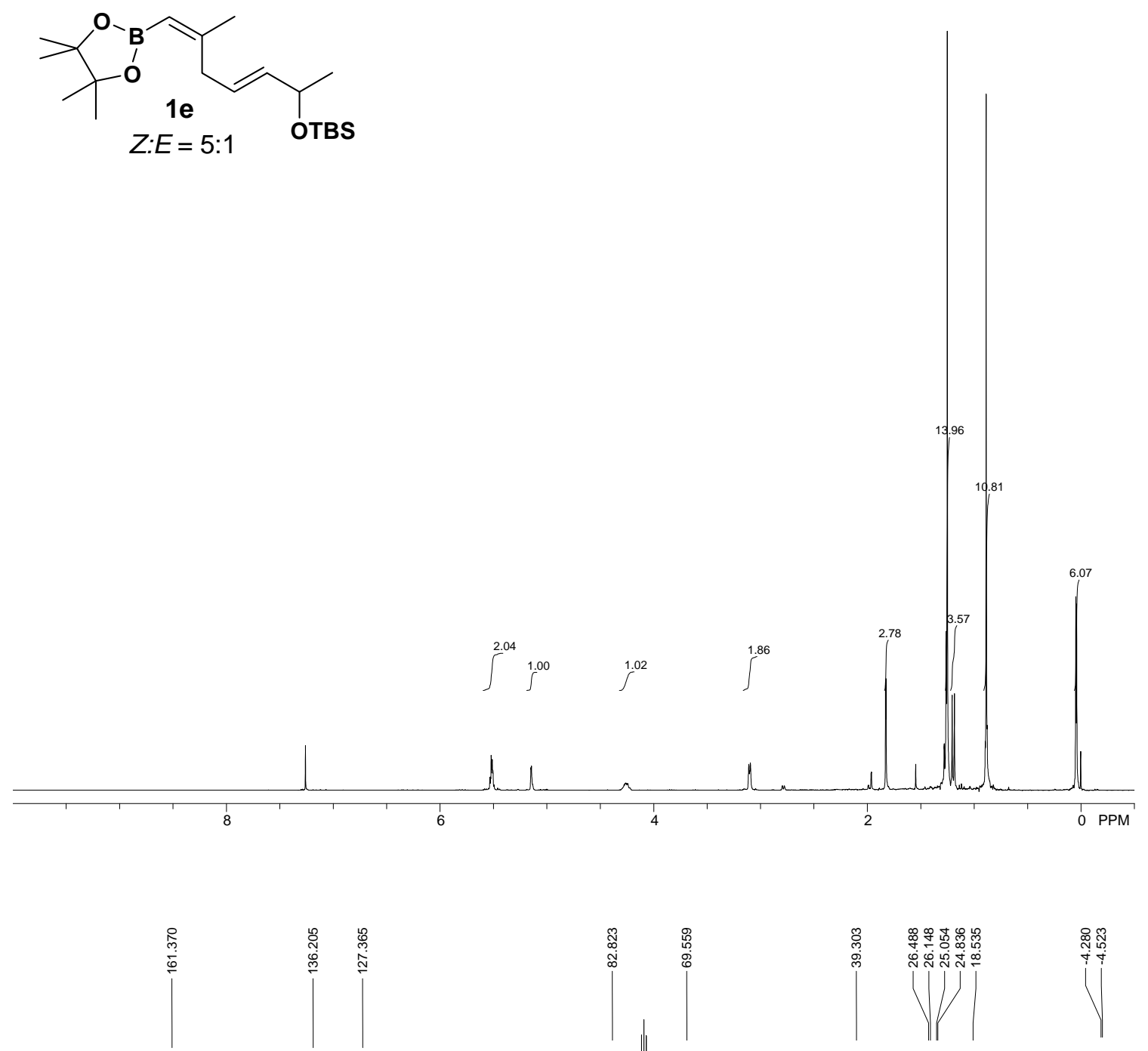

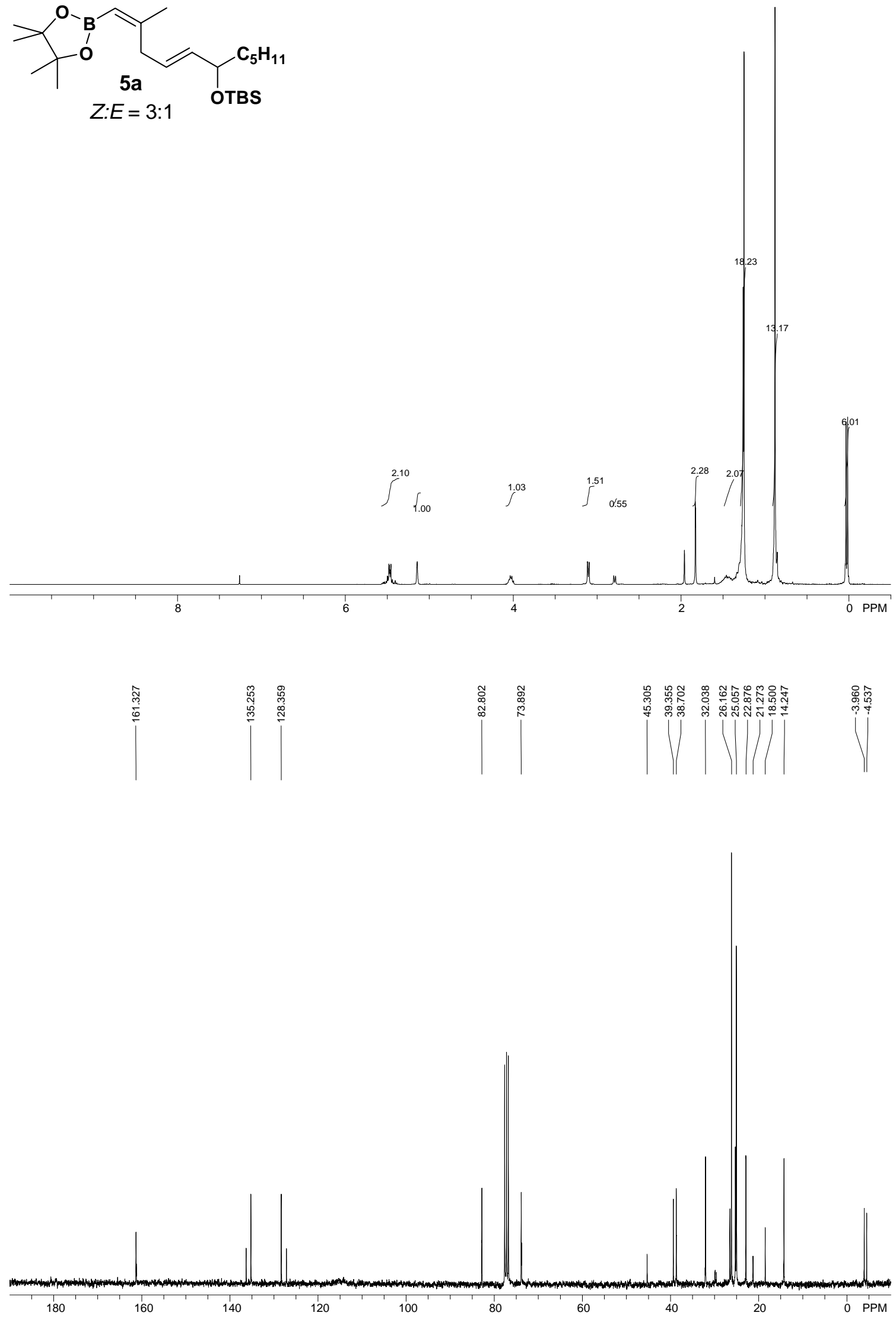

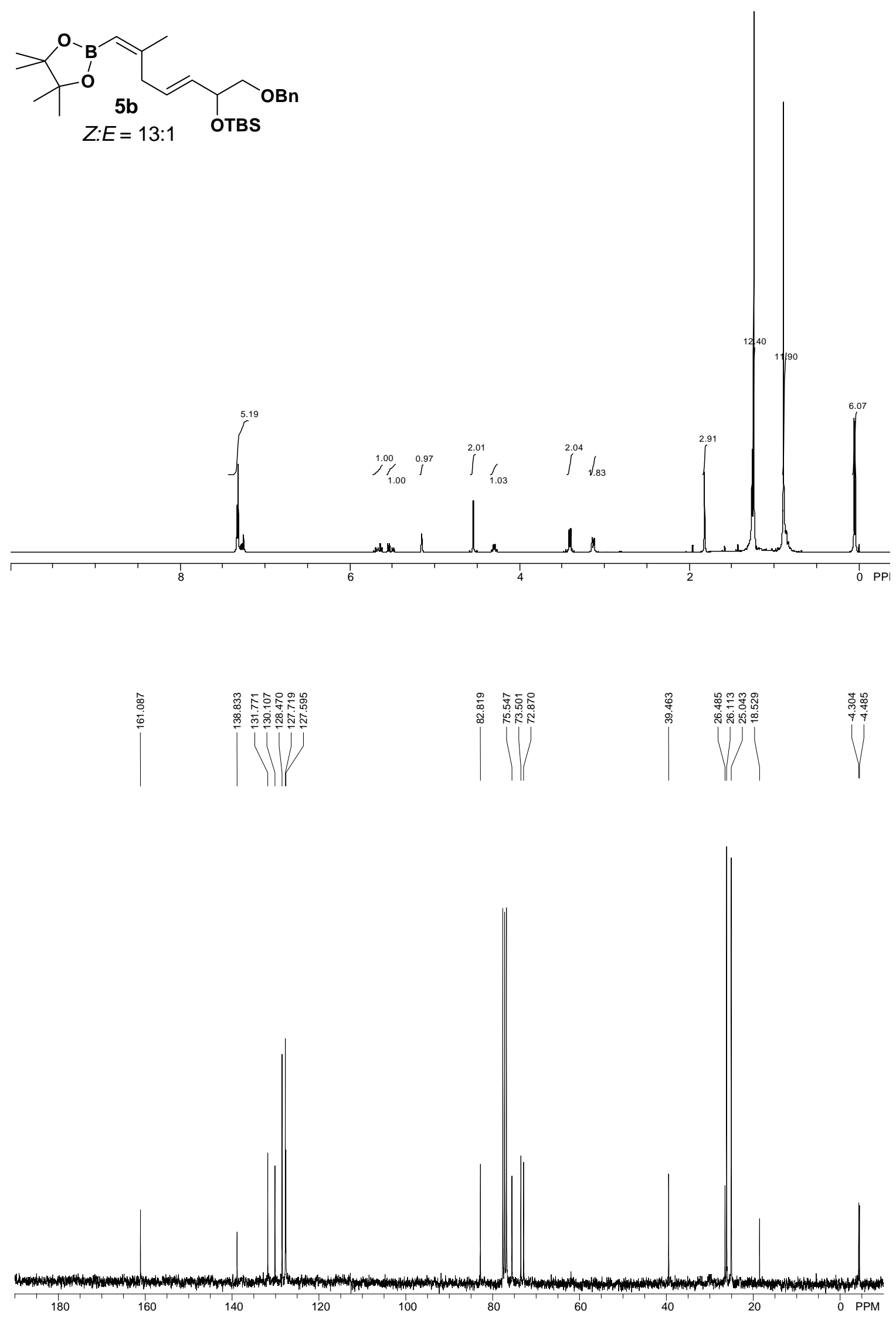

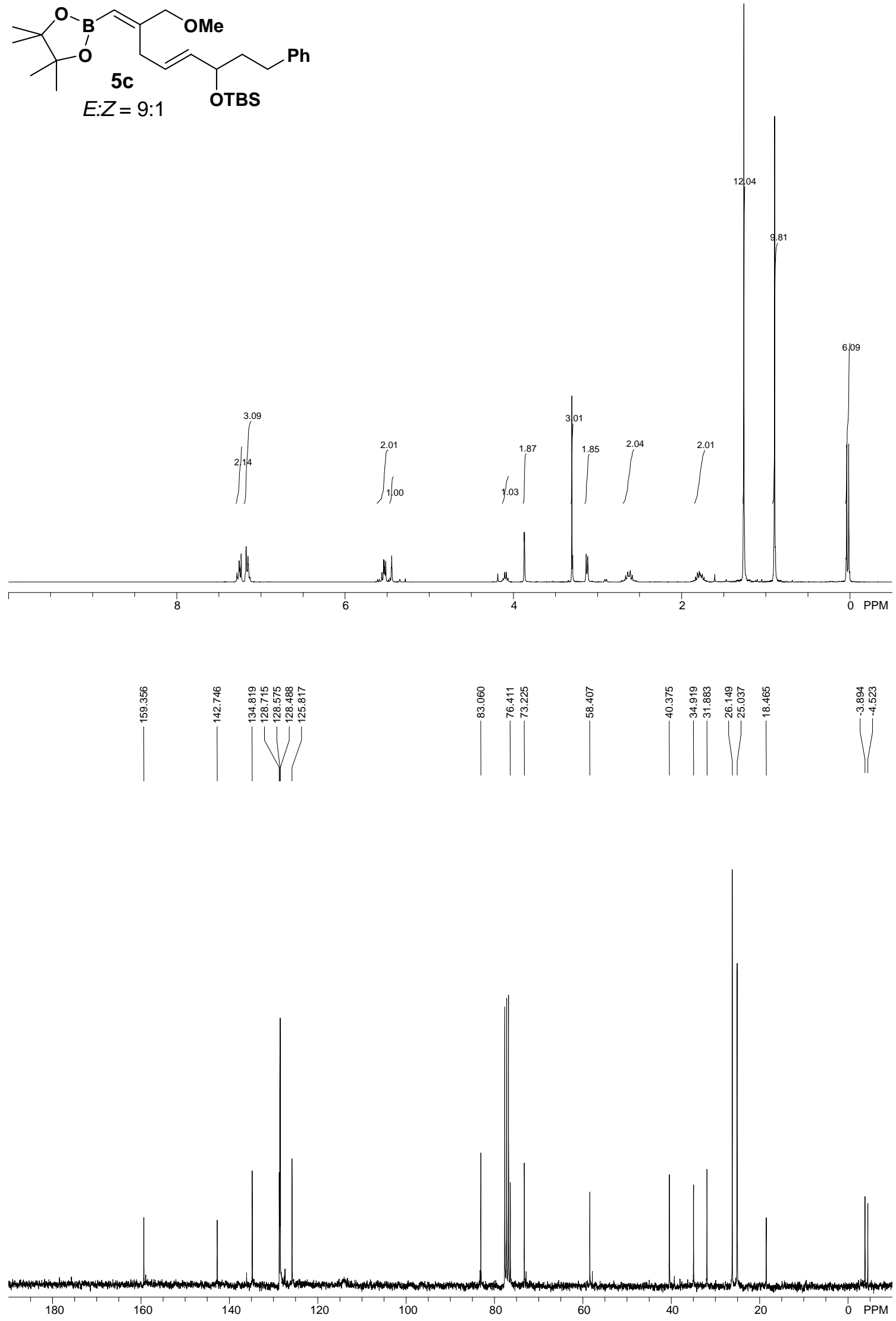

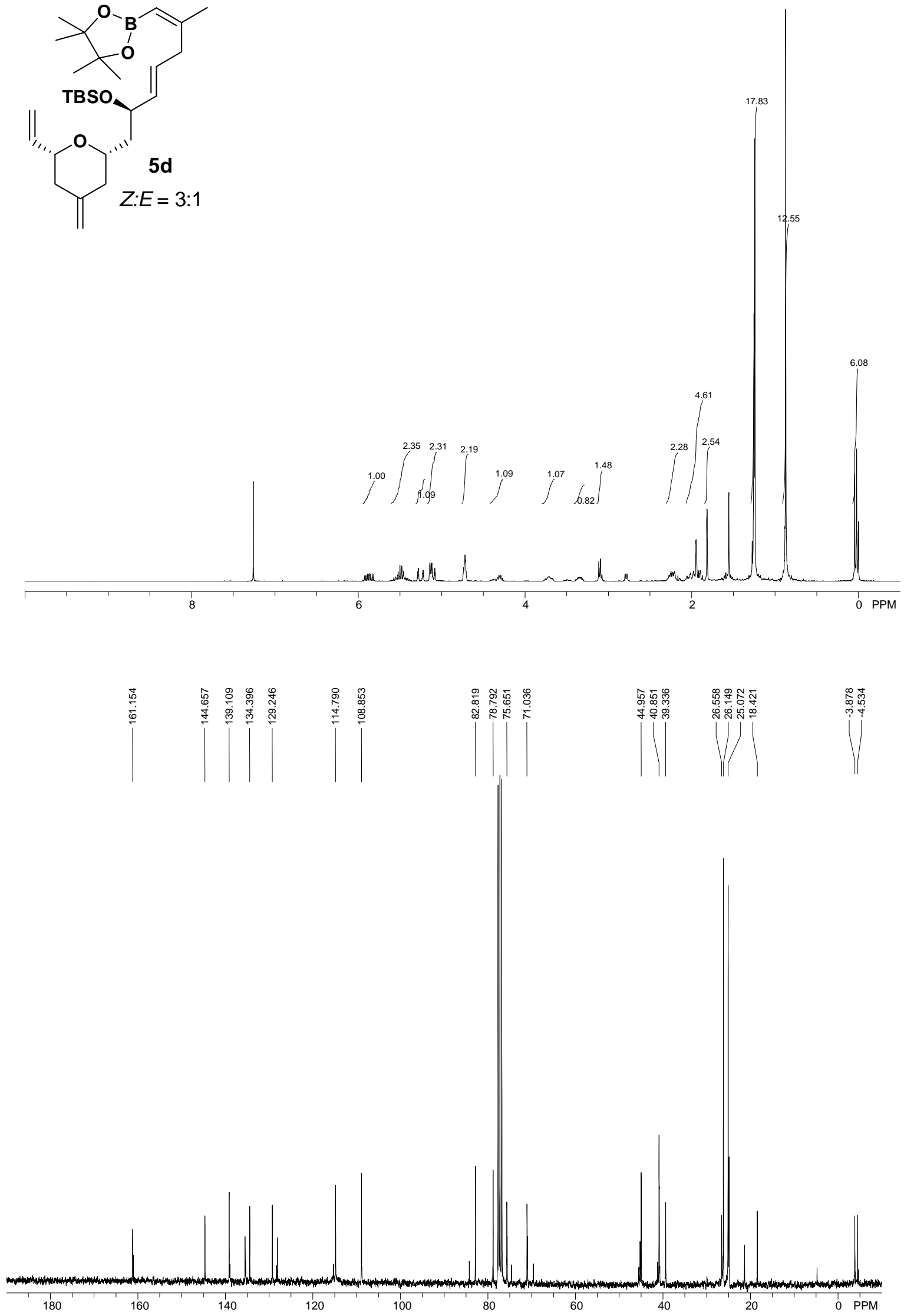
<smiles>C/C=C/C(O)C/C(C)=C\C=C/C(=O)OCC</smiles>
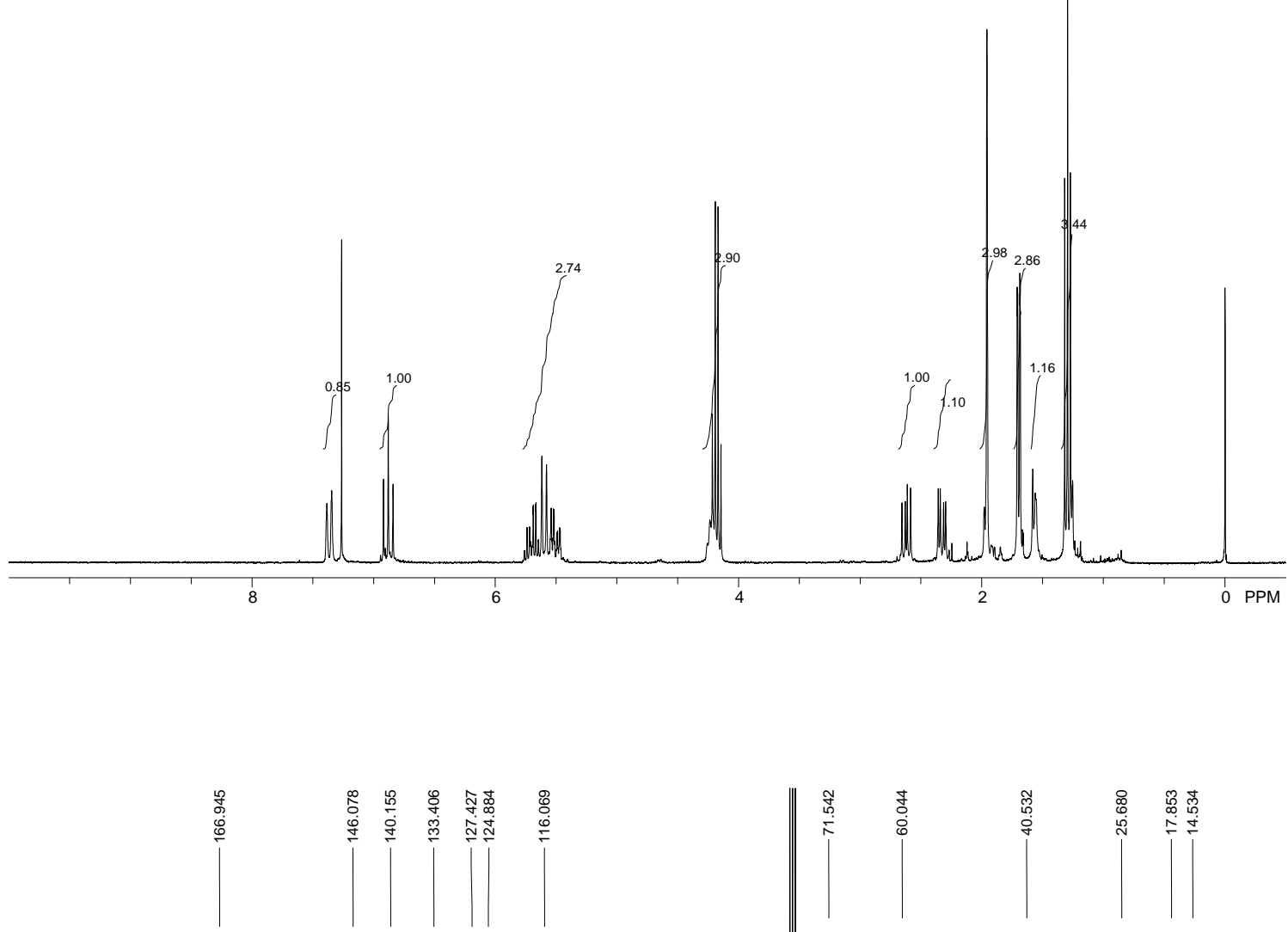

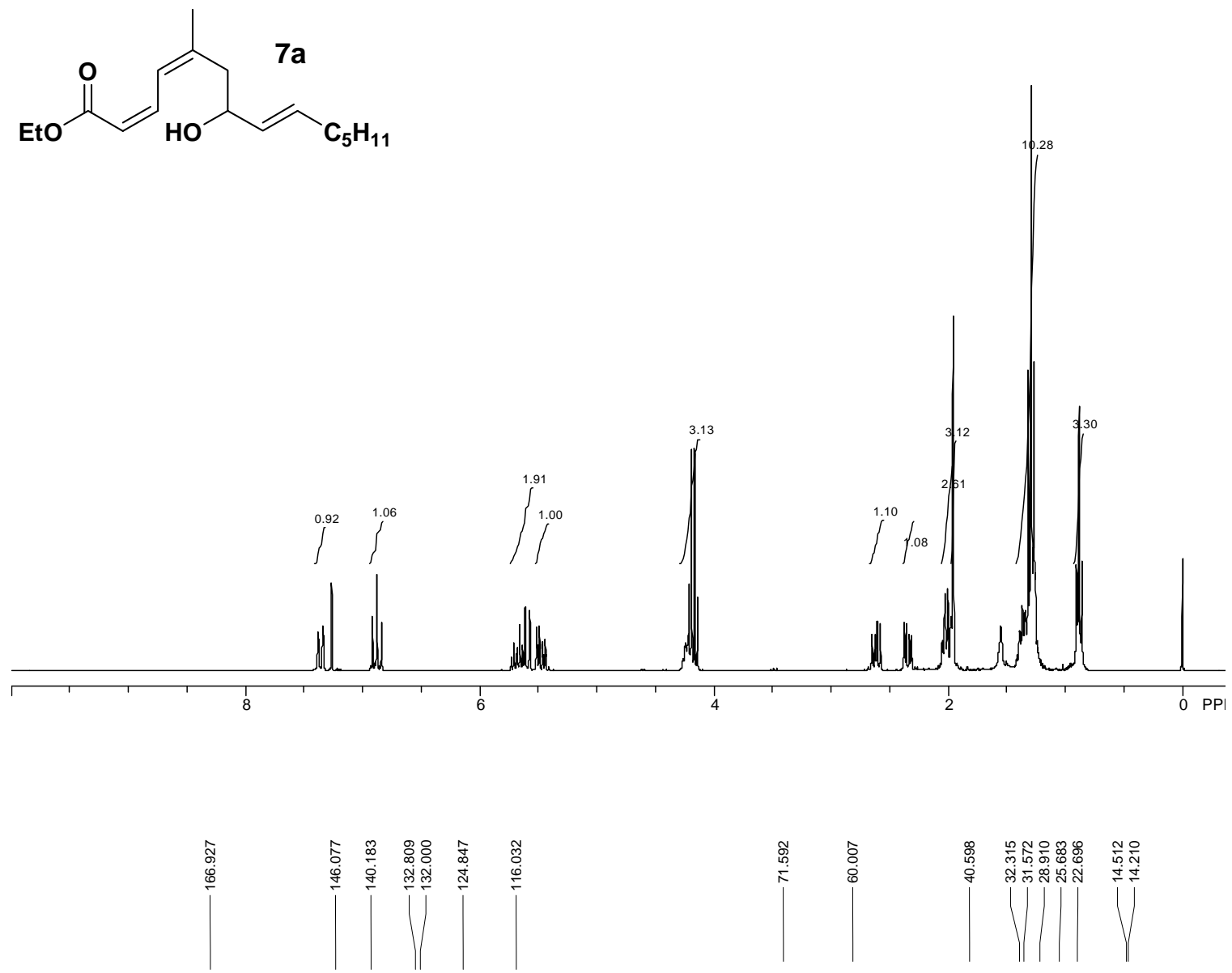

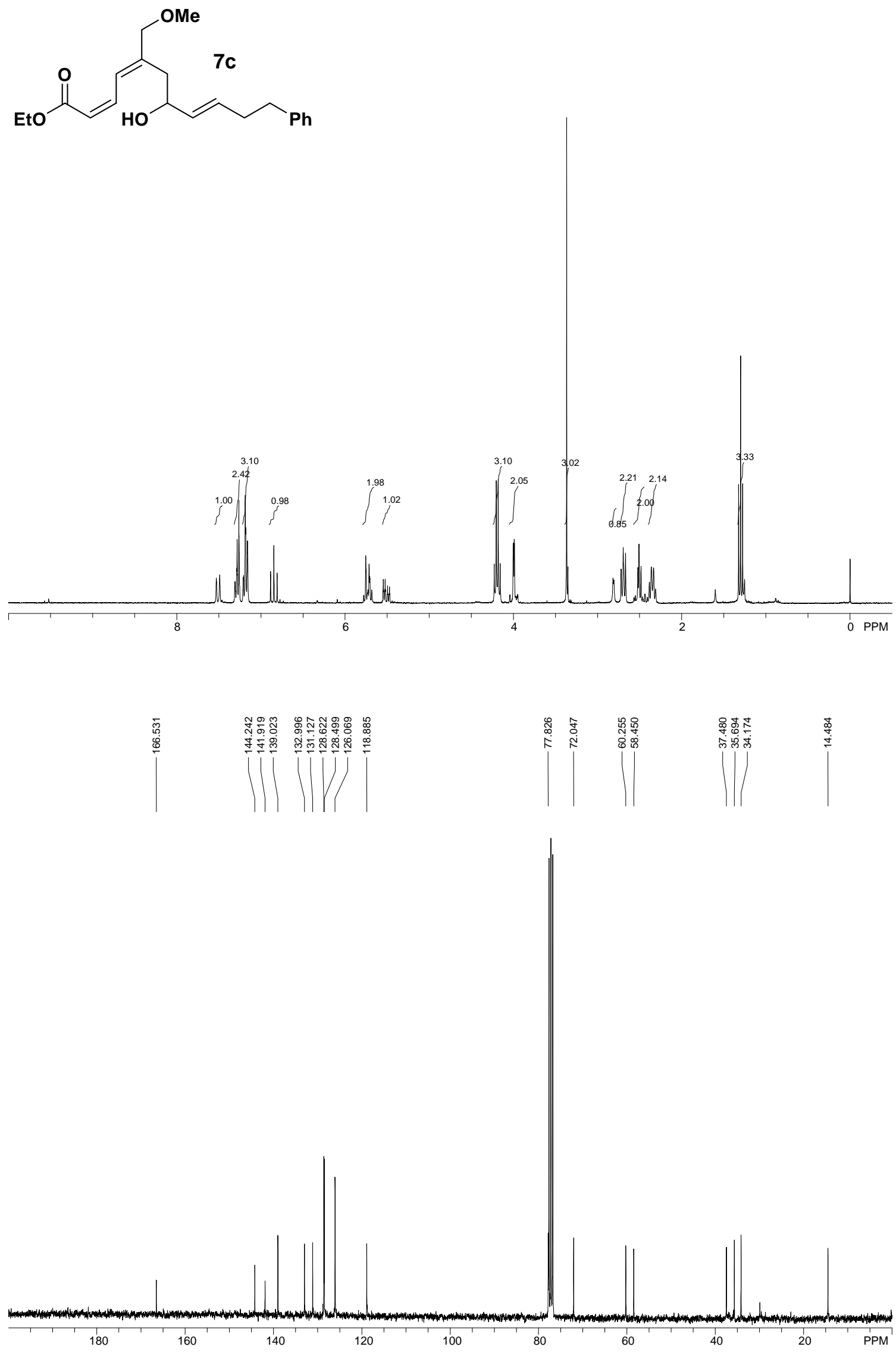
<smiles>CCOC(=O)/C=C\C=C(\C)CC(O)/C=C/c1ccccc1</smiles>
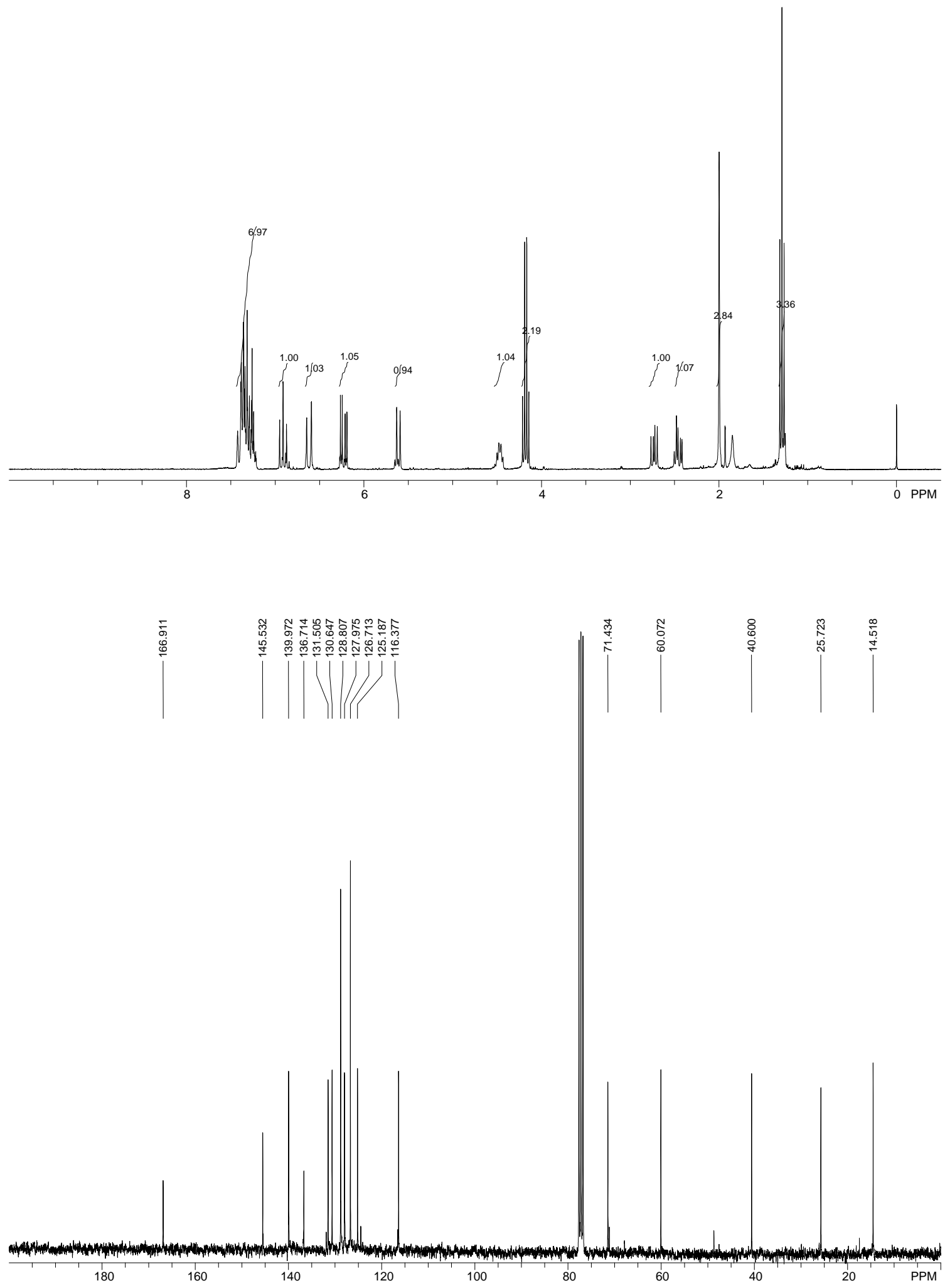

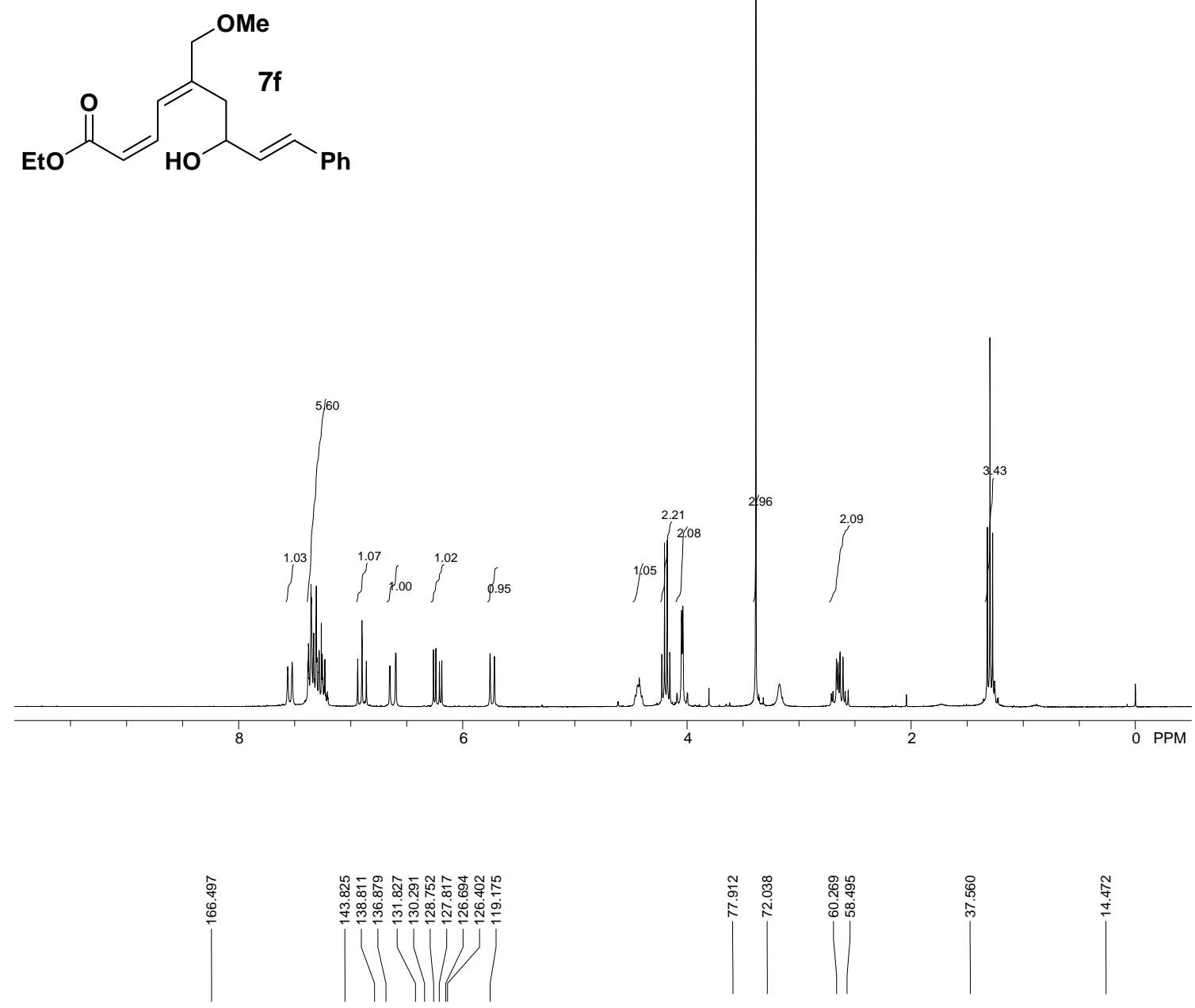

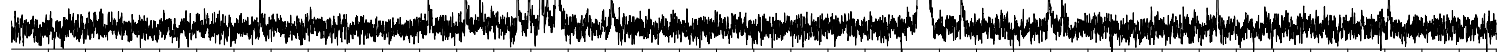

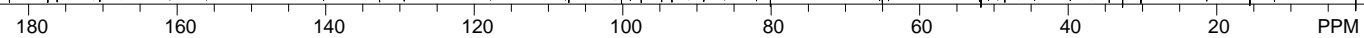


<smiles>C=CC[C@H](CCc1ccccc1)OC(=O)C(c1ccccc1)(c1ccccc1)C(F)(F)F</smiles>

Mosher's ester for homoallylic alcohol leading to $5 \mathrm{c}$<smiles>CCOC(=O)/C=C\C=C(/COC)CC(/C=C/CCc1ccccc1)OC(=O)[C@@](C)(OC)[C@@](C)(c1ccccc1)C(F)(F)F</smiles>

Mosher's ester for rac-7c

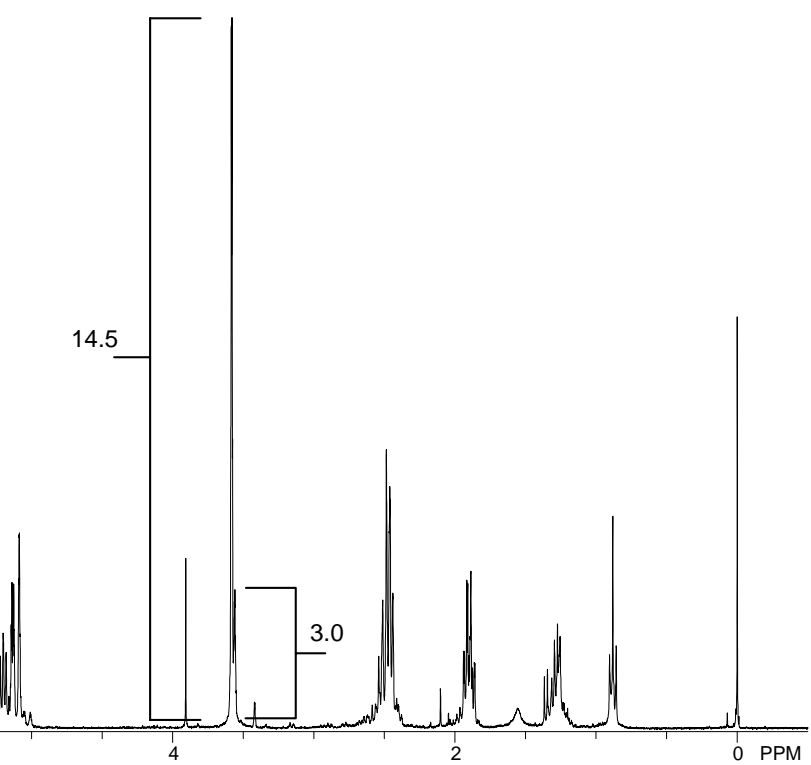

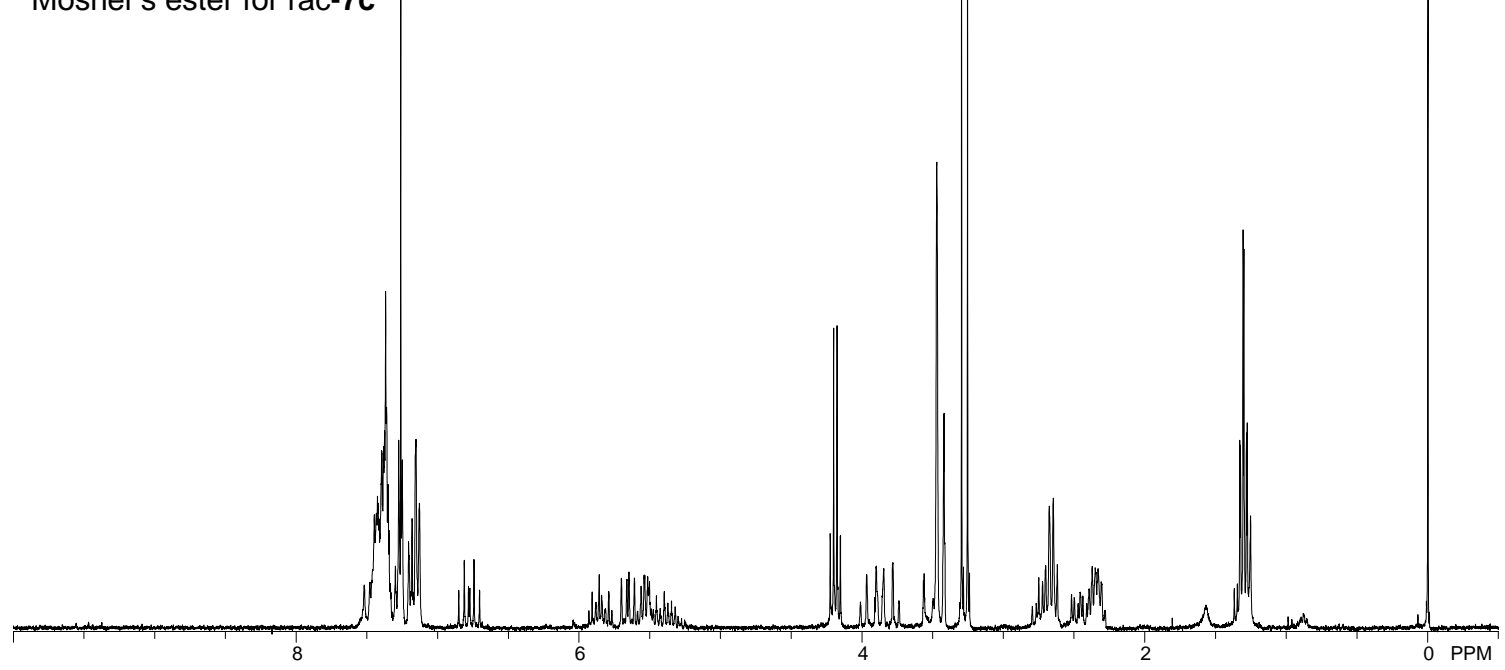



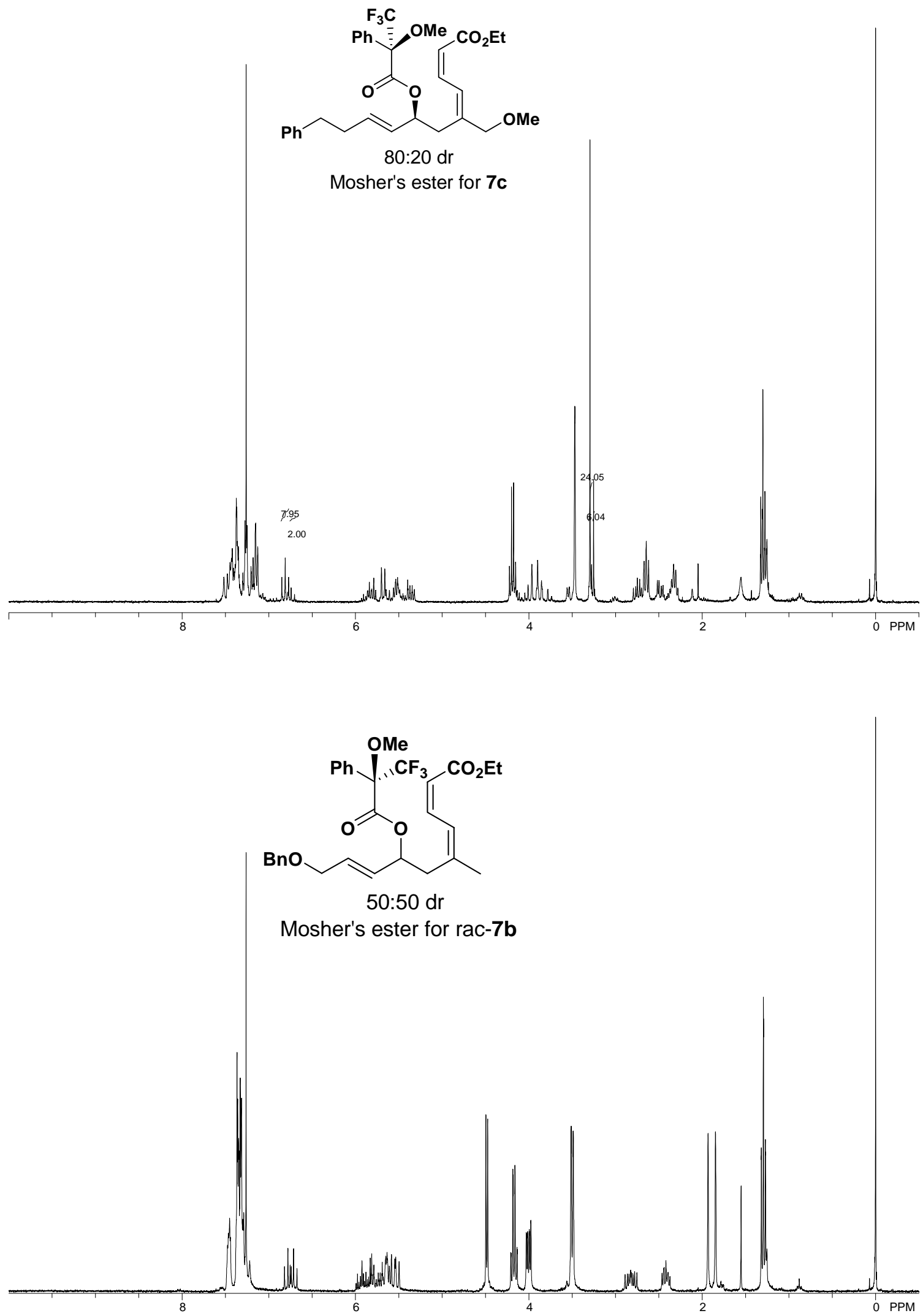


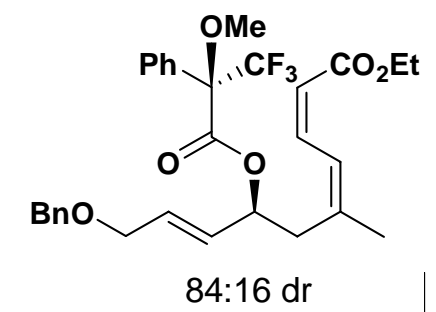

Mosher's ester for $\mathbf{7 b}$

(rearrangement in $\mathrm{CH}_{2} \mathrm{Cl}_{2}$ )
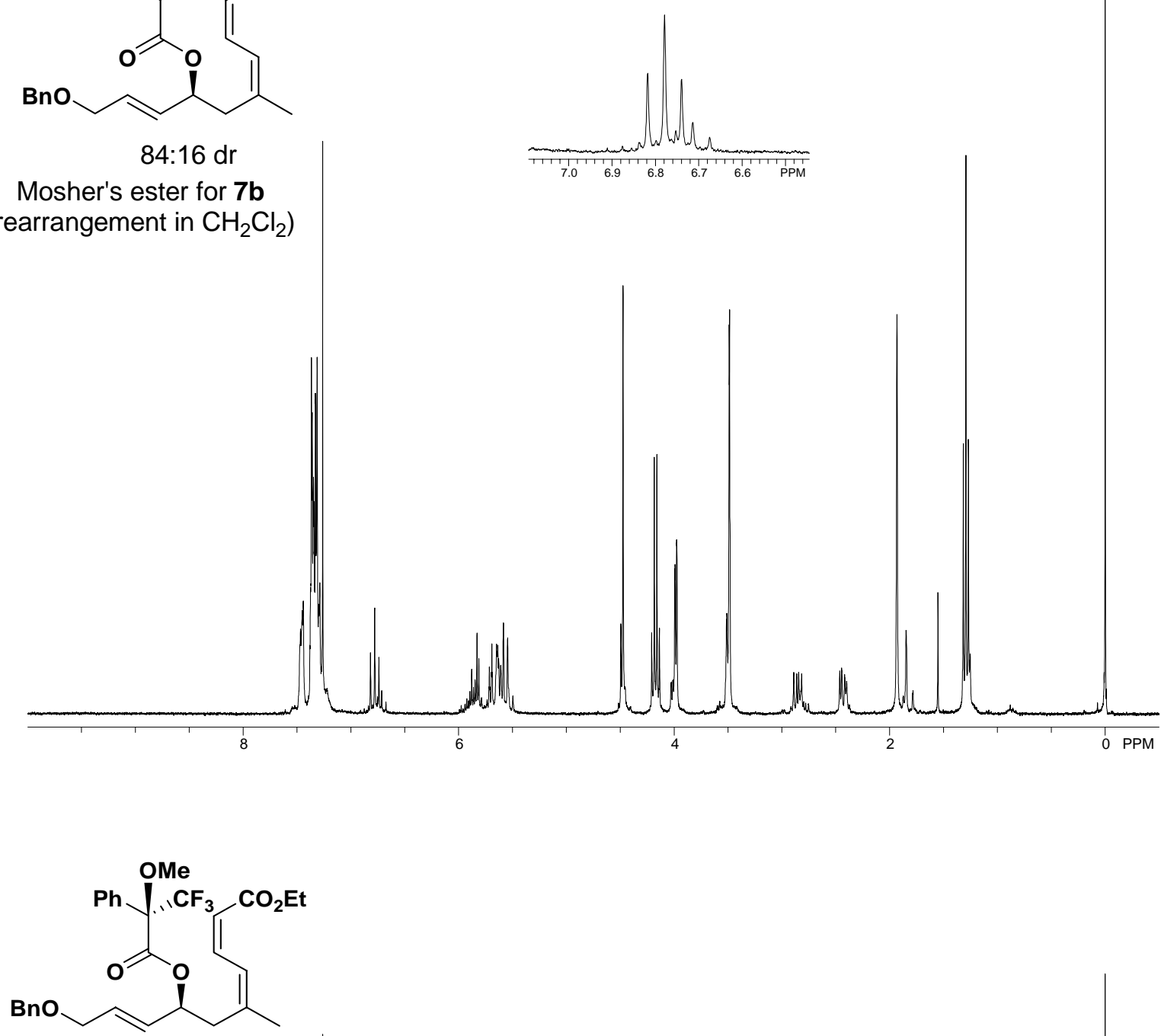

single diasteriomer Mosher's ester of $\mathbf{7 b}$ (rearrangement in $\mathrm{Et}_{2} \mathrm{O}$ )

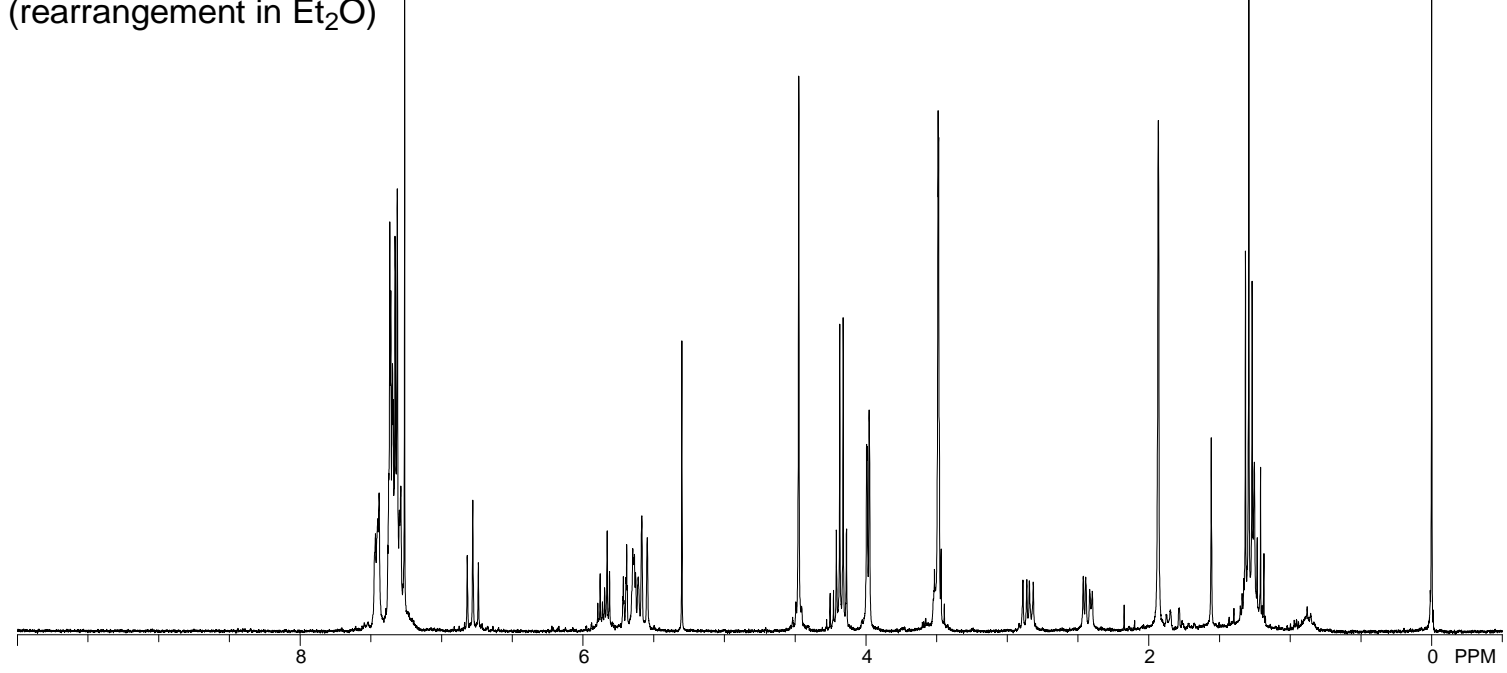



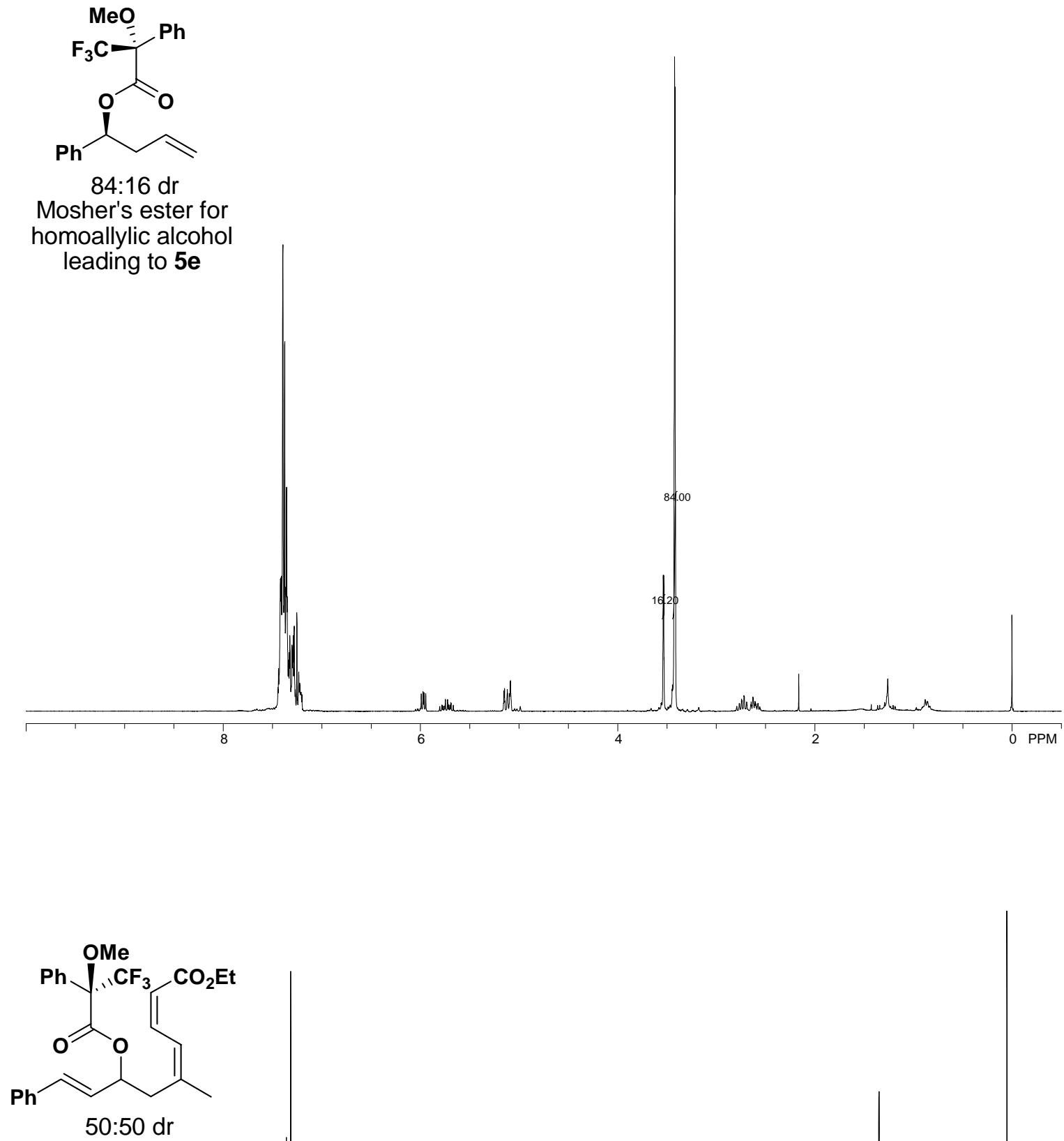

Mosher's ester for 7e

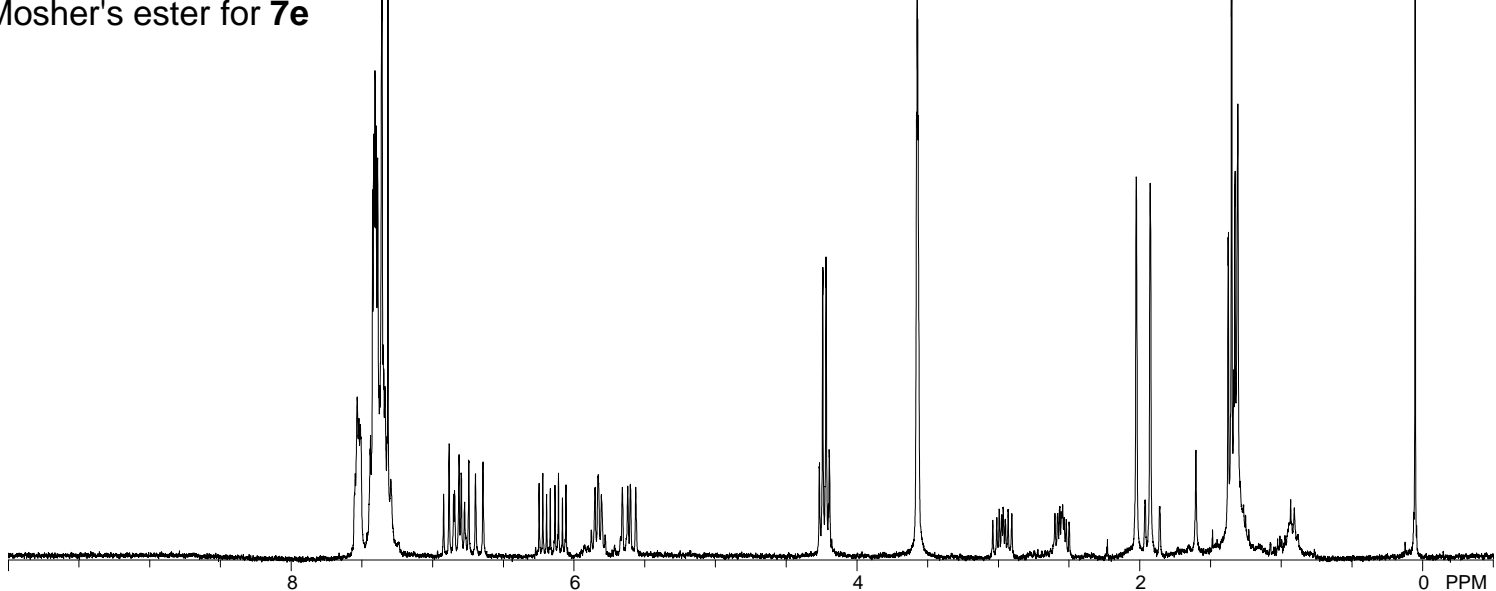

